\title{
Validity of ROX index in prediction of risk of intubation in patients with COVID-19 pneumonia
}

\begin{abstract}
Introduction: One important concern during the management of COVID-19 pneumonia patients with acute hypoxemic respiratory failure is early anticipation of the need for intubation. ROX is an index that can help in identification of patients with low and those with high risk of intubation. So, this study was planned to validate the diagnostic accuracy of the ROX index for prediction of COVID-19 pneumonia outcome (the need for intubation) and, in addition, to underline the significant association of the ROX index with clinical, radiological, demographic data.

Material and methods: Sixty-nine RT-PCR positive COVID-19 patients were enrolled. The following data were collected: medical history, clinical classification of COVID-19 infection, the ROX index measured daily and the outcome assessment.

Results: All patients with severe COVID-19 infection (100\%) were intubated (50\% of them on the $3^{\text {rd }}$ day of admission), but only $38 \%$ of patients with moderate COVID-19 infection required intubation (all of them on the $3^{\text {rd }}$ day of admission). The ROX index on the $1^{\text {st }}$ day of admission was significantly associated with the presence of comorbidities, COVID-19 clinical classification, CT findings and intubation ( $p \leq 0.001$ for each of them). Regression analysis showed that sex and ROX.1 are the only significant independent predictors of intubation [AOR (95\% CI): 16.9 (2.4- 117), $0.77(0.69-0.86)$ ], respectively. Cut-off point of the ROX index on the $1^{\text {st }}$ day of admission was $\leq 25.26(90.2 \%$ of sensitivity and $75 \%$ of specificity).

Conclusions: ROX is a simple noninvasive promising tool for predicting discontinuation of high-flow oxygen therapy and could be used in the assessment of progress and the risk of intubation in COVID-19 patients with pneumonia.
\end{abstract}

Key words: ROX index, COVID-19, intubation risk

Adv Respir Med. 2021; 89: 1-7

\section{Introduction}

Severe hypoxemia resulting from COVID-19 pneumonia is often associated with near normal respiratory system compliance, which is almost never seen in severe ARDS. However, COVID-19 pneumonia in most cases falls under the Berlin definition of ARDS [1, 2].

Severely hypoxemic patients with COVID-19 pneumonia, despite sharing a single etiology, may have different presentations: markedly dyspneic or normally breathing ("happy" hypoxemia); and either responsive to prone position or not. So, the same disease presents itself with impressive heterogenity. Two primary "phenotypes": type L, characterized by low elastance (i.e., high compliance), low recruitment and low lung weight and type $\mathrm{H}$, characterized by high elastance, high recruitment and high lung weight were reported [3]. For patients with COVID-19 infection, oxygen supplementation via low-flow nasal cannula may be sufficient, however; in patients with acute hypoxemic respiratory failure, higher flow of oxygen may be needed, and noninvasive modalities (HFNC and NIV) may be used rather than proceeding directly to intubation [4]. A systematic review from July 2020 identified one trial evaluating HFNC in patients with COVID-19, which suggested that it reduced the need for mechanical ventilation and improvements in oxygenation compared with standard oxygen therapy [5]. The ROX in-

Address for correspondence: Heba Wagih Abdelwahab, Mansoura University, Mansoura, Egypt; e-mail: wagihheba84@gmail.com 
dex defined as the ratio of oxygen saturation as measured by pulse oximetry/ $/ \mathrm{FiO}_{2}$ to respiratory rate can help identify those patients with low and those with high risk of intubation [6,7]. One important concern during the management of COVID-19 pneumonia patients with acute hypoxemic respiratory failure is not to delay intubation. So, this study was planned to validate the diagnostic accuracy of the ROX index for COVID-19 pneumonia outcome (the need for intubation) and, in addition, to underline the significant association of the ROX index with clinical, radiological, and demographic data.

\section{Material and methods}

This diagnostic study was conducted on 69 RT-PCR positive COVID-19 patients with radiological evidence of pneumonia attending different quarantine places in Egypt from April 2020 to June 2020. Mild COVID-19 patients (presenting with respiratory symptoms without radiological evidence of pneumonia), individuals with chronic respiratory failure and those with conditions that affect pulse oximeter reading (e.g. nail polish) were excluded. This study was conducted within the required ethics guidelines of Mansoura institutional research board ethics committee (code number: R.20.05.832) and approved by the Ministry of Health and Population. Training and research sector (REC) (code: Com. NO/Dec.No:14-2020/2).

The following data were collected:

- medical history, e.g. age, sex, occupation, comorbidities, previous treatment;

- COVID-19 infection clinical classification according to the Ministry of Health and Population in Egypt:

- moderate case: the presence of symptoms suggestive of COVID-19 infection with radiological evidence of pneumonia;

- severe case: moderate case who meet any of the following: oxygen saturation < $93 \%$, respiratory rate $\geq 30$ breath $\backslash \mathrm{min}$ at rest and patients with $>50 \%$ pulmonary lesion progression within 24 to $48 \mathrm{~h}$;

- radiological data:

- pulmonary computerized tomography (CT) pattern of COVID-19 [3]: a) type L COVID-19 pneumonia - subpleural and along the lung fissures ground-glass densities with moderately increased lung weight; b) type H COVID-19 pneumonia - the increased amount of non-aerated tissue;
- the ROX index refers to combination of the ratio of oxygen saturation (as measured by pulse oximetry) to fraction of inspired oxygen and respiratory rate $\left(\mathrm{SpO}_{2} / \mathrm{FiO}_{2} / \mathrm{RR}\right)$. It was calculated daily for all patients who underwent oxygen therapy from the measured value of $\mathrm{SpO}_{2}$ and respiratory rates (breaths/ $/ \mathrm{min})$ and supplemental oxygen $\left(\mathrm{FiO}_{2}\right.$ values $)$ $[6,7]$;

- other data indicate severity: $\mathrm{PO}_{2} / \mathrm{FiO}_{2}, \mathrm{SpO}_{2} /$ $/ \mathrm{FiO}_{2}$, other organ failure, d-dimer, serum ferritin, the presence of shock;

- all patients were primarily evaluated for the need for oxygen therapy and follow-up for one week for either clinical improvement (fever drops, better respiratory symptoms, hemodynamics stability, less need for oxygen $(<0.4)$ or deterioration with the necessity for invasive mechanical ventilation. Mechanical ventilation was considered in the presence of worsening or persistent respiratory distress, respiratory rate more than 40 breaths/min, $\mathrm{SpO}_{2}$ less than $90 \%$ despite maximum oxygen flow and $\mathrm{FiO}_{2}$, acidemia with $\mathrm{pH}$ less than 7.25 , significant hemodynamic instability and multiorgan failur;

- failure of oxygen therapy was considered when the patient needed invasive mechanical ventilation within one week from the beginning of treatment with oxygen.

\section{Statistical analysis of data}

The collected data was prepared, tabulated, and statistically analyzed using statistical package for social science (SPSS) version 16. Frequencies and percentages were used to present nominal variables, while means (SD), or median (min-max) were used to present continuous data according to the results of Shapiro-Wilk testing of normality of variables. Some variables were calculated: the ROX index using the formula $\left(\mathrm{SpO}_{2} / \mathrm{FiO}_{2}\right) /$ respiratory rate as ROX.1:ROX value on the first day of admission, ROX.2:ROX value on the $2^{\text {nd }}$ day of admission, ROX.3:ROX value on the $3^{\text {rd }}$ day of admission. Modified ROX was calculated using the formula: $\left(\mathrm{PO}_{2} / \mathrm{FiO}_{2}\right) /$ respiratory rate. Significance testing was done with the help of chi-square test, and the independent-samples Mann-Whitney $\mathrm{U}$ test for categorical, non-parametric data, respectively. Also, Spearman correlation was used to test an association between non-parametric data. Multivariate logistic regression was done to determine the independent significant predictors of intubation. Variables found to have a significant association with the intubation outcome (intubat- 
ed/not) in univariate analysis were entered in the regression model. Receiver operator curve (ROC) analysis was used to determine the most accurate cut-off point for prediction of intubation. The level of significance was set at $p<0.05$.

\section{Results}

The study included 69 patients (mean age was 53 years old). About $78.3 \%$ of them were males. Most of the studied patients had no comorbidity (56.5\%). Hypertension was the most common comorbidity (24.6\%) reported. Seventy percent of them had CT of Type L, and 65\% of them were classified clinically as having moderate COVID-19 infection (Table 1, Figure 1). About $59 \%$ of the studied patients were intubated. At intubation, median (min-max) of ROX, modified ROX and $\mathrm{PO}_{2} / \mathrm{FiO}_{2}$ ratio was 3.88 (3.33-6.09), 5 (3.14-5.52), 90.9 (60-109.09), respectively.

ROX 1, 2, 3 indices were significantly associated with intubation ( $\mathrm{p} \leq 0.001$ for each of them). COVID-19 clinical classification was significantly associated with intubation $(\mathrm{p} \leq 0.001)$. All patients with severe COVID-19 infection $(100 \%)$ were intubated (Table 2), however, only $38 \%$ of moderate COVID-19 infection patients who needed oxygen therapy were intubated. All subjects with moderate COVID-19 infection were intubated on the $5^{\text {th }}$ day of admission, but $50 \%$ of patients with severe COVID-19 infection were intubated on the $3^{\text {rd }}$ day of admission (Table 2, Figure 1).

Table 1. Clinical characteristics of the patients $(n=69)$

\begin{tabular}{|c|c|c|c|}
\hline Parameter & & $\mathbf{N}$ & $\%$ \\
\hline Age mean (SD) & $53.3(8.7)$ & & \\
\hline \multirow[t]{2}{*}{ Sex } & Male & 54 & 78.3 \\
\hline & Female & 15 & 21.7 \\
\hline \multirow[t]{5}{*}{ Comorbidity } & No & 39 & 56.5 \\
\hline & IDDM & 6 & 8.7 \\
\hline & HTN & 11 & 15.9 \\
\hline & IHD/HTN & 6 & 8.7 \\
\hline & IHD & 7 & 10.1 \\
\hline \multirow{2}{*}{$\begin{array}{l}\text { COVID clinical } \\
\text { classification }\end{array}$} & Moderate & 45 & 65.2 \\
\hline & Sever & 24 & 34.8 \\
\hline \multirow[t]{2}{*}{ CT type } & $\mathrm{L}$ & 48 & 69.6 \\
\hline & $\mathrm{H}$ & 21 & 30.4 \\
\hline \multirow[t]{2}{*}{ Intubation } & Non intubated & 28 & 40.6 \\
\hline & Intubated & 41 & 59.4 \\
\hline \multirow{3}{*}{$\begin{array}{l}\text { Day of intubation } \\
(\mathrm{n}=41)\end{array}$} & $3^{\text {rd }}$ from admission & 12 & 29.3 \\
\hline & $4^{\text {th }}$ from admission & 6 & 14.6 \\
\hline & $5^{\text {th }}$ from admission & 23 & 56.1 \\
\hline
\end{tabular}

HTN — hypertension; IDDM — insulin-dependent diabetes mellitus; IHD ischaemic heart disease

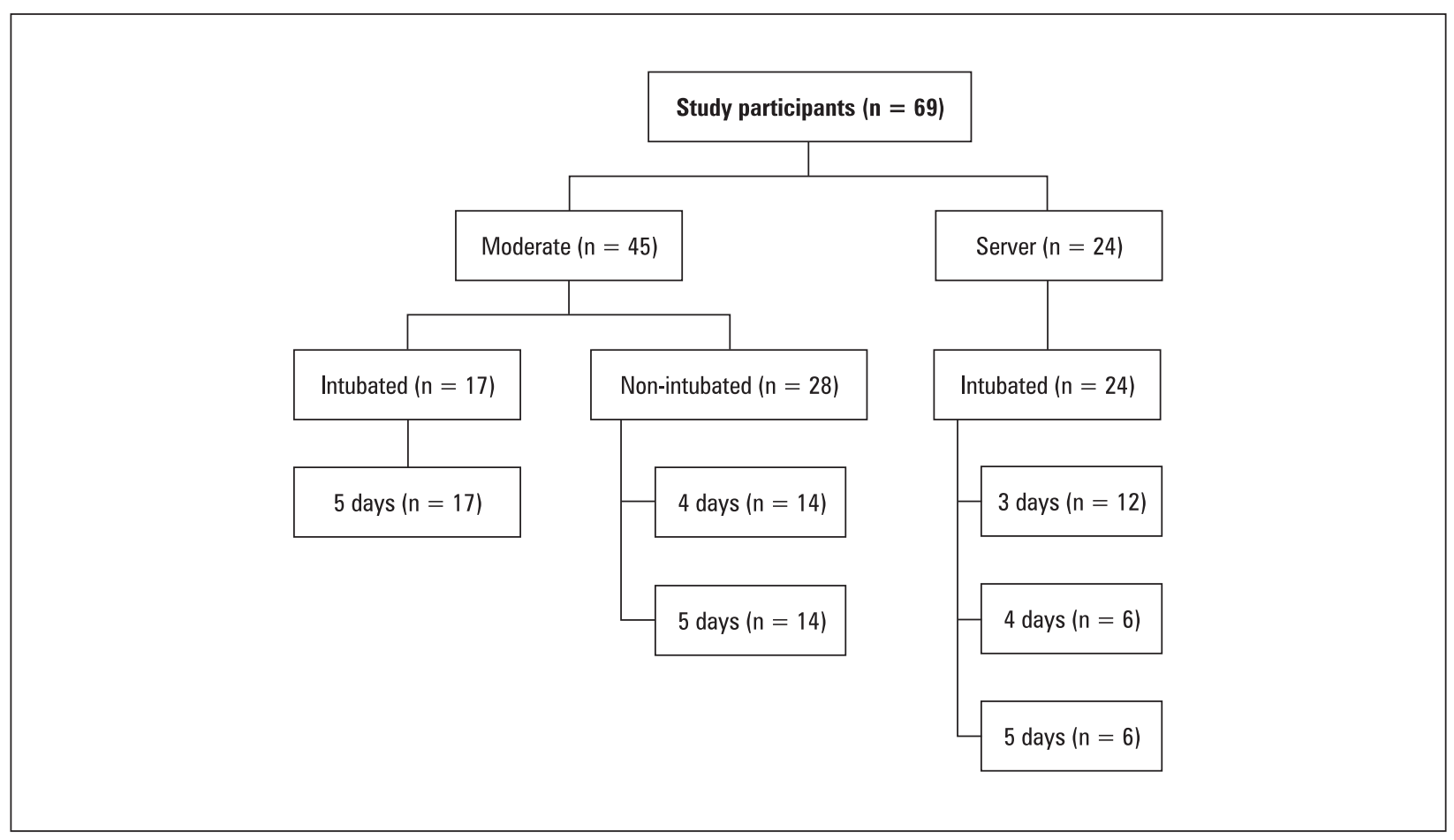

Figure 1. The days spent until day of endotracheal intubation or improvement in non-intubated patients 
Table 2. Risk factors of intubation among the patients with COVID-19 ( $\mathrm{n}=41$ )

\begin{tabular}{|c|c|c|c|c|c|}
\hline \multicolumn{2}{|l|}{ Parameter } & Total $\mathbf{n}$ & $\begin{array}{c}\text { Non intubated } \\
\mathbf{n}(\%)\end{array}$ & $\begin{array}{c}\text { Intubated } \\
n=41 \\
n(\%)\end{array}$ & Significance \\
\hline \multicolumn{2}{|l|}{ Age median (min-max) } & & $54(38-67)$ & $52(39-67)$ & $Z=-0582,0: 0.561^{*}$ \\
\hline \multirow[t]{2}{*}{ Sex } & Male & 54 & $26(48.1)$ & $28(51.9)$ & $X 2: 5.7, p=0.017$ \\
\hline & Female & 15 & $2(13.3)$ & $13(86.7)$ & \\
\hline \multirow[t]{2}{*}{ Comorbidity } & Absence & 39 & $21(53.8)$ & $18(46.2)$ & $X 2: 6.5, p=0.011$ \\
\hline & Presence & 30 & $7(23.3)$ & $23(76.7)$ & \\
\hline \multirow[t]{2}{*}{ COVID-19 classification } & Moderate & 45 & $28(62.2)$ & $17(37.8)$ & $X 2: 25.1, p \leq 0.001$ \\
\hline & Sever & 24 & 0 & $24(100)$ & \\
\hline \multirow[t]{2}{*}{ CT pattern } & Type L & 48 & $28(58.3)$ & $20(41.7)$ & $X 2: 20.6, p \leq 0.001$ \\
\hline & Type H & 21 & 0 & $21(100)$ & \\
\hline \multicolumn{2}{|l|}{ ROX.1 median (min-max) } & & $26.3(16.4-28.6)$ & $10.6(8.8-26.6)$ & $Z:-5.59, p \leq 0.001^{*}$ \\
\hline \multicolumn{2}{|l|}{ ROX.2 median (min-max) } & & $26.6(12.8-28.6)$ & $7.8(7.3-25.8)$ & $Z:-5.58, p \leq 0.001^{*}$ \\
\hline \multicolumn{2}{|l|}{ ROX.3 median (min-max) } & & $26.6(12.8-28.9)$ & $7.5(4.8-18.9)$ & $Z:-6.69, p \leq 0.001^{*}$ \\
\hline \multicolumn{2}{|l|}{ ROX.4 median (min-max) } & & $28.6(26.3-28.9)$ & $6.9(3.3-7.9)$ & $\mathrm{Z}:-6.52, \mathrm{p} \leq 0.001^{*}$ \\
\hline \multicolumn{2}{|l|}{ ROX.5 median (min-max) } & & $28.3(28.27-28.57)$ & $3.8(3.7-4.4)$ & $Z:-5.04, p \leq 0.001^{*}$ \\
\hline \multicolumn{2}{|c|}{ Lymphocyte median (min-max) } & & $1400(1100-2100)$ & $1250(1000-2100)$ & $Z:-2.38, p=0.017^{*}$ \\
\hline \multicolumn{2}{|l|}{ WBCs median (min-max) } & & $7000(3400-11000)$ & 7600 (3400-12000) & $Z: 1.34, p=0.180^{*}$ \\
\hline \multicolumn{2}{|c|}{ Platelet median (min-max) } & & $130000(86000-960000)$ & $130000(87000-160000)$ & $Z:-0.47, p=0.637^{*}$ \\
\hline \multicolumn{2}{|c|}{ Creatinine median (min-max) } & & $0.9(0.7-1.4)$ & $0.9(0.5-1.5)$ & $Z:-1.02, p=0.307^{*}$ \\
\hline \multicolumn{2}{|c|}{ Albumin median (min-max) } & & $3.3(2.8-4.1)$ & $3.4(2.7-4)$ & $Z: 0.259, p=0.796^{*}$ \\
\hline \multicolumn{2}{|c|}{ Ferritin median (min-max) } & & $770(120-1300)$ & $800(220-1700)$ & $Z: 1.15, p=0.25^{*}$ \\
\hline \multicolumn{2}{|c|}{ D dimer median (min-max) } & & $980(400-1500)$ & $1000(430-2100)$ & $Z: 1.49, p=0.134^{*}$ \\
\hline
\end{tabular}

Also, sex, comorbidities, CT pattern, lymphocyte absolute count were significantly associated with intubation $(\mathrm{p}=0.017,0.011, \leq 0.001, \leq$ $0.001,0.017$ ) (Table 2). However, the results of multivariate logistic regression of predictors of intubation among COVID-19 patients showed that female sex and ROX.1 were the only significant independent predictors of intubation [AOR (95\% CI): 16.9 (2.4-117), 0.77 (0.69-0.86)], $p=0.004$, $\leq 0.001$, respectively (Table 3 ).

ROX.1 was significantly associated with the presence of comorbidities, COVID-19 clinical classification, CT findings, intubation $(\mathrm{p} \leq$ 0.001 for each of them). Also, there was a negative significant association with albumin $(\mathrm{r}=-0.276$, $\mathrm{p}=0.022$ ) (Table 4).

The results of ROC analysis for ROX 1, 2, 3 as predictors of intubation have shown (AUC, p value): $(0.897, \leq 0.001),(0.896, \leq 0.001),(0.967$, $\leq 0.001$ ), respectively. Cut-off points of ROX.1, ROX.2, and ROX.3 were $\leq 25.26$ (90.2\% of sensitivity and $75 \%$ of specificity), $\leq 21.34$ (90\% of sen- sitivity and $75 \%$ of specificity). and $\leq 11.71$ (90\% of sensitivity and $100 \%$ of specificity) (Table 5).

\section{Discussion}

Delayed intubation has been shown to be associated with poor clinical outcome, so predicting the failure of noninvasive ventilation or oxygen therapy has remained an important area of research [7]. One important concern during the management of COVID-19 pneumonia is not to delay intubation in patients with acute hypoxemic respiratory failure. An objective method to identify subjects who are likely to fail to respond to oxygen therapy is needed. The ROX index is remarkably simple and has the potential to become a routine parameter in clinical practice.

Roca and colleagues [6, 7] first published an index ROX which can predict whether the patient will fail to use high frequency nasal canula (HFNC) in pneumonia in ICU, and they reported that a ROX value of $>4.88$ predicted the success 
Table 3. Multivariate logistic regression of predictors of intubation among COVID-19 patients $(\mathrm{n}=69$ )

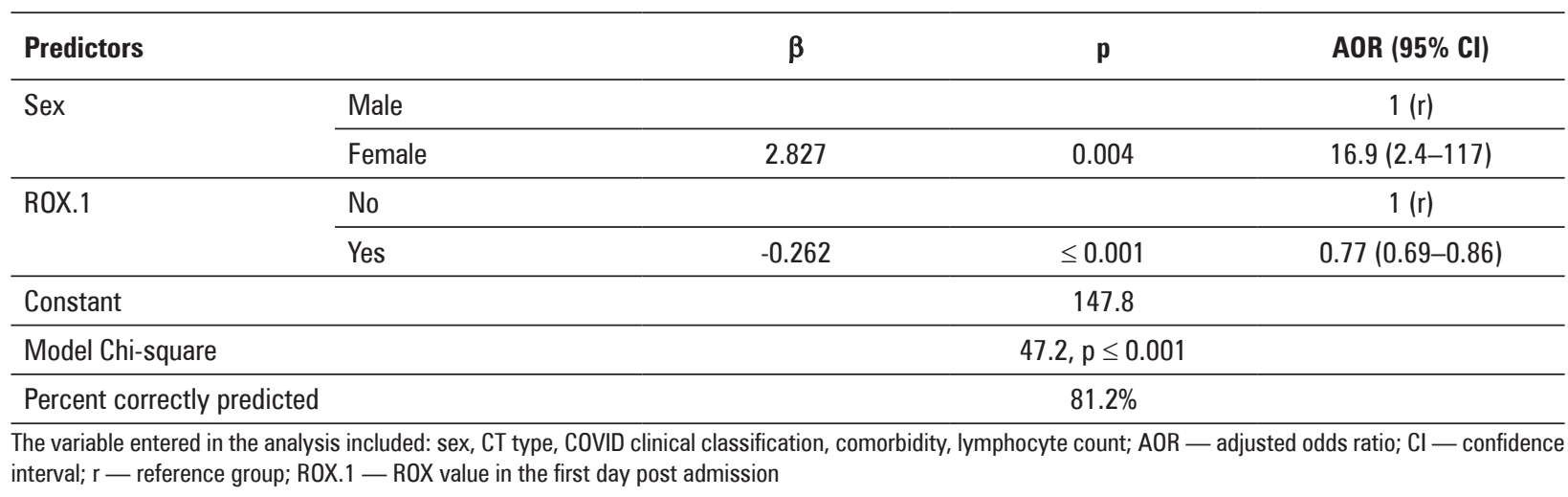

Table 4. Association of ROX.1 with other parameters of patients

\begin{tabular}{|c|c|c|c|}
\hline & & Median (min-max) & Significance \\
\hline \multirow[t]{2}{*}{ Sex } & Male & $16.4(8.7-28.6)$ & Z: $-0.86, \mathrm{p}: 0.389^{*}$ \\
\hline & Female & $24.8(8.8-28.6)$ & \\
\hline \multirow[t]{2}{*}{ Comorbidities } & No & $25.4(8.8-28.6)$ & Z: $-3.17, \mathrm{p}: 0.002^{*}$ \\
\hline & Yes & $15.6(8.8-26.6)$ & \\
\hline \multirow[t]{2}{*}{ COVID classification } & Moderate & $25.4(15.6-28.6)$ & $Z:-6.82, p \leq 0.001^{*}$ \\
\hline & Sever & $9.7(8.8-10.6)$ & \\
\hline \multirow[t]{2}{*}{ CT } & Low & $25.4(10.5-28.6)$ & $Z:-5.26, p \leq 0.001^{*}$ \\
\hline & High & $8.9(8.8-25.2)$ & \\
\hline \multirow[t]{2}{*}{ Intubation } & No & $26.3(16.4-28.6)$ & $Z:-5.59, \mathrm{p} \leq 0.001^{*}$ \\
\hline & Yes & $10.6(8.8-26.6)$ & \\
\hline \multicolumn{2}{|c|}{ Lymphocyte absolute count } & $r=0.06, p: 0.592^{* *}$ & \\
\hline \multicolumn{2}{|l|}{ WBCs } & $r=-0.09, p: 0.462^{* *}$ & \\
\hline \multicolumn{2}{|l|}{ Platelet } & $r=-0.021, p: 0.865^{* *}$ & \\
\hline \multicolumn{2}{|l|}{ Creatinine } & $r=0.202, p: 0.096^{* *}$ & \\
\hline \multicolumn{2}{|l|}{ Albumin } & $r=-0.276, p: 0.022^{* *}$ & \\
\hline \multicolumn{2}{|l|}{ Ferritin } & $r=-0.071, p: 0.563^{* *}$ & \\
\hline \multicolumn{2}{|l|}{ D dimer } & $r=-0.187, p: 0.124^{* *}$ & \\
\hline
\end{tabular}

*Independent-Samples Mann-Whitney U test; ${ }^{*}$ Spearman's Rho

of HFNC. Also, in Nicholas and Robin's study [8], the ROX index was validated in 191 critically ill patients enrolled at 5 centers in France and Spain, and the ROX index score of 4.88 was used as predictive of outcomes. The area under the curve at 12 hours of HFNC use was 0.752. Both studies calculated the ROX index at 0, 2, 6, 12 hours from the onset of oxygen therapy.

Rodriguez et al. [9] found that the ROX index in critically ill patients in ICU was higher in the subjects who were successfully separated from HFNC at the first trial than in those who failed (12.7 vs 10.2, $p=0.002$ ). The ROX index $\geq 9.2$ predicted successful separation from HFNC at the first trial (specificity of $50 \%$, sensitivity of $84 \%$, positive predictive value of $93 \%$, negative predictive value of $30 \%$, and accuracy of $80 \%$ ). They calculated the ROX index up to 48 hours.

All previous studies were conducted on critically illpatients other than COVID-19. To the best of our knowledge, there is only one recent study done by Belz and coworkers [10] using the ROX index for monitoring of oxygen therapy by HFNC in a SARS-CoV-2 severe pneumonia admitted to ICU with proven COVID-19, and the authors found that performance characteristics of ROX 
Table 5. Validity of ROX indices in prediction of intubation

\begin{tabular}{lccccccc}
\hline Parameter (cut off point) & P value & Sensitivity & Specificity & PPV & NPV & AUC & 95\% Cl \\
\hline ROX.1 $\leq 25.26$ & $\leq 0.001$ & $90.2 \%$ & $75 \%$ & $84.1 \%$ & $84 \%$ & 0.897 & $(0.824-0.970)$ \\
ROX.2 $\leq 21.34$ & $\leq 0.001$ & $90 \%$ & $75 \%$ & $84.1 \%$ & $84 \%$ & 0.896 & $(0.819-0.974)$ \\
ROX.3 $\leq 11.71$ & $\leq 0.001$ & $90 \%$ & $100 \%$ & $100 \%$ & $87.5 \%$ & 0.976 & $(0.947-1.004)$ \\
\hline
\end{tabular}

AUC — area under the curve; $\mathrm{Cl}$ - confidence interval; NPV — negative predictive value; PPV — positive predictive value; ROX.1 — ROX value in the first day post admission; ROX.2 - ROX value in the $2^{\text {nd }}$ day post admission

at 0.5 hour using the previous published cut-off value of 4.88 by Roca and colleagues [6, 7] had a $81 \%$ sensitivity and a $38 \%$ specificity.

In this study, we used the ROX index as a simple noninvasive tool in COVID-19 pneumonia patients for prediction of the need for intubation. In contrast to previous studies, the ROX index was measured daily as we collected data from different medical facilities that dealt with COVID-19 cases, and we found that all patients with severe COVID-19 infection (100\%) were intubated (Table 2), however, only $38 \%$ of moderate COVID-19 infection patients who needed oxygen therapy were intubated. All persons with moderate COVID-19 infection were intubated on the $5^{\text {th }}$ day of admission but $50 \%$ of patients with severe COVID-19 infection were intubated on the $3^{\text {rd }}$ day of admission.

The results of multivariate logistic regression of predictors of intubation among COVID-19 patients have shown that female sex and ROX.1 (the ROX value on the first day of admission) are the only significant independent predictors of intubation in this study. Cut-off point of ROX.1 (the ROX value on the $1^{\text {st }}$ day of admission) was $\leq 25.26(90.2 \%$ of sensitivity and $75 \%$ of specificity) (AUC, p): (0.897, $\leq 0.001$ ), ROX.2 (the ROX value on the $2^{\text {nd }}$ day of admission) was $\leq 21.34$ (90\% of sensitivity and $75 \%$ of specificity) and of ROX.3 (the ROX value on the $3^{\text {rd }}$ day of admission) $\leq 11.71(90 \%$ of sensitivity and $100 \%$ of specificity). The cut-off value is higher than in other studies because we conducted this study on a heterogeneous cases of different severities as we involved some less severe cases of moderately severe COVID-19 pneumonia. ROX.1 was significantly associated with the presence of comorbidities, COVID-19 clinical classification, CT findings, intubation ( $\mathrm{p} \leq 0.001$ for each of them). Also, there was a negative significant association with albumin.

Clinicians could use the ROX index to assess progress in COVID-19 patients, making serial measurements and incorporating it when considering decisions to increase care. During the $1^{\text {st }}$ day of admission, scores below the cut-offs given in this study would prompt anticipation of the need for earlier intubation. Once the $1^{\text {st }}$ day point is reached, a score $>25.26$ increases clinician confidence that the patient will succeed.

\section{Conclusions}

ROX is a simple noninvasive promising tool for predicting discontinuation of high-flow oxygen therapy and could be used by clinicians in the assessment of progress and the risk of intubation in COVID-19 patients with pneumonia. Further studies on a large number of COVID-19 patients would be necessary to support our results.

\section{Conflict of interest}

None declared.

\section{References:}

1. Zhu Na, Zhang D, Wang W, et al. A novel coronavirus from patients with pneumonia in China, 2019. N Engl J Med. 2020; 382(8): 727-733, doi: 10.1056/NEJMoa2001017, indexed in Pubmed: 31978945.

2. Ranieri MV, Rubenfeld GD, Thompson BT, et al. Acute respiratory distress syndrome: the Berlin Definition. JAMA 2012; 307(23): 2526-2533, doi: 10.1001/jama.2012.5669, indexed in Pubmed: 22797452.

3. Gattinoni L, Chiumello D, Caironi P, et al. COVID-19 pneumonia: different respiratory treatments for different phenotypes? Intensive Care Med. 2020; 46(6): 1099-1102, doi: 10.1007/ s00134-020-06033-2, indexed in Pubmed: 32291463.

4. Anesi LG. Coronavirus disease 2019 (COVID-19): Critical care and airway management issues. UptoDate. Available online: https://www.uptodate.com/contents/coronavirus-disease-2019covid-19-critical-care-and-airway-management-issues/print. [Last accessed at: 16 July 2020].

5. Rochwerg B, Solo K, Darzi A, et al. Update alert: ventilation techniques and risk for transmission of coronavirus disease, including COVID-19. Ann Intern Med. 2020; 173(6): W122, doi: 10.7326/L20-0944, indexed in Pubmed: $\underline{32735446}$.

6. Roca O, Messika J, Caralt B, et al. Predicting success of highflow nasal cannula in pneumonia patients with hypoxemic respiratory failure: The utility of the ROX index. J Crit Care. 2016; 35: 200-205, doi: 10.1016/j.jcrc.2016.05.022, indexed in Pubmed: 27481760 .

7. Roca O, Caralt B, Messika J, et al. An index combining respiratory rate and oxygenation to predict outcome of nasal high-flow therapy. Am J Respir Crit Care Med. 2019; 199(11): 1368-1376, doi: 10.1164/rccm.201803-05890C, indexed in Pubmed: 30576221 
8. Hill NS, Ruthazer R. Predicting outcomes of high-flow nasal cannula for acute respiratory distress syndrome. An index that ROX. Am J Respir Crit Care Med. 2019; 199(11): 1300-1302, doi: 10.1164/rccm.201901-0079ED, indexed in Pubmed: 30694696.

9. Rodriguez M, Thille AW, Boissier F, et al. Predictors of successful separation from high-flow nasal oxygen therapy in patients with acute respiratory failure: a retrospective mono- center study. Ann Intensive Care. 2019; 9(1): 101, doi: 10.1186/ s13613-019-0578-8, indexed in Pubmed: 31511996.

10. Blez D, Soulier A, Bonnet F, et al. Monitoring of high-flow nasal cannula for SARS-CoV-2 severe pneumonia: less is more, better look at respiratory rate. Intensive Care Med. 2020; 46(11): 2094-2095, doi: 10.1007/s00134-020-06199-9, indexed in Pubmed: $\underline{32737522}$. 